\title{
Análisis de la evolución de la panificación en la provincia del Carchi como patrimonio alimentario a través de la revalorización de sus técnicas y preparaciones tradicionales
}

\section{Analysis of the evolution of baking in the province of Carchi as food heritage through the revaluation of its techniques and traditional preparations}

Msc. Daniel Gonzalo Fierro Mosquera.

Msc Hugo Jonathan Herrera Jiménez.

Universidad Tecnológica Equinoccial, Ecuador

Autor para correspondencia: daniel.fierro@ute.edu.ec, hugo.herrera@ute.edu.ec

Fecha de recepción: 20 de julio de 2018 - Fecha de aceptación: 30 de agosto de 2018

Resumen: El pan es un alimento que se ha constituido como una de las principales fuentes de energía, formando parte de la dieta en diferentes lugares del mundo durante la historia, llegando a ser recomendado por la organización mundial de la salud (OMS), que considera adecuado un consumo de 200-250 g/día. La panificación del Ecuador es diversa e invaluable en cada región y representa una tradición gastronómica arraigada en los pueblos desde que el trigo llego a América y se lo transformó en pan. Muchas variedades endémicas de pan han perdurado en el tiempo conservando sus características y aún forman parte de celebraciones tradicionales. El objetivo que pretende esta investigación, es determinar las características culturales de la panificación del Carchi, a través del análisis histórico destacando sus utensilios, técnicas de preparación que hacen de estas únicas en el Ecuador, como contribución al conocimiento y valoración de los productos tradicionales del patrimonio alimentario del Ecuador. La metodología es cualitativa fundamentada en una interacción social no ofensiva, basada en una revisión histórica cultural de fuentes primarias y secundarias, así como de aplicaciones experimentales de procesos gastronómicos y de estandarización, direccionadas a la revalorización gastronómica, cultural, de esta parte del patrimonio alimentario. Como resultado de la investigación, se pretende contribuir a la revalorización de la panificación ecuatoriana, como elemento importante del patrimonio alimentario, a través de la construcción de un documento que fundamente su estructura en narraciones, lazos culturales, reseñas, equipamiento tradicional, preparaciones considerando la importancia que tiene el consumo de pan en gran parte de las provincias del Ecuador.

Palabras clave: panes del Ecuador; características históricas culturales; procedimientos tradicionales; patrimonio alimentario y utensilios tradicionales

Abstract: Bread is a food that has been constituted as one of the main sources of energy, being part of the diet in different places of the world during the history, getting to be recommended by the world organization of the health (OMS), that considers adequate a consumption of 200-250 g / day. The baking of Ecuador is diverse and invaluable in each region and represents a gastronomic tradition rooted in the villages since the wheat arrived in America and transformed it into bread. Many endemic varieties of bread have survived over time while retaining their characteristics and still form part of traditional celebrations. The objective of this research is to determine the cultural 
characteristics of Ecuadorian bread making, through the historical and productive analysis of representative loaves from different provinces, as a contribution to the knowledge and valuation of traditional products of Ecuador's food heritage. The methodology is qualitative based on a nonoffensive social interaction, based on a historical cultural review of primary and secondary sources, as well as experimental applications of gastronomic processes and standardization, aimed at the cultural and gastronomic revaluation of this part of the food heritage. As a result of the research, it aims to contribute to the revaluation of Ecuadorian bread making, as an important element of heritage, through the construction of a document that bases its structure on narrations, cultural ties, reviews, traditional equipment used and recipes linked to Each region, considering the importance of the consumption of bread in much of the provinces of Ecuador.

Key words: Ecuadorian bread; cultural historical characteristics; traditional procedures; food heritage and traditional utensils

\section{Introducción}

El Ministerio de Cultura y Patrimonio tiene como prioridad enfocarse en la revalorización de la gastronomía ecuatoriana y pretende salvaguardar la diversidad de productos; por ende, la panadería de Ecuador se enfoca en el producto, historia, técnicas, procesos, ingredientes y recetas, ya que todos estos temas son de interés fundamental para el patrimonio alimentario de los ecuatorianos.

El Ministerio de Cultura y Patrimonio tiene como prioridad enfocarse en la revalorización de la gastronomía ecuatoriana y pretende salvaguardar la diversidad de productos; por ende, la panadería de Ecuador se enfoca en el producto, historia, técnicas, procesos, ingredientes y recetas, ya que todos estos temas son de interés fundamental para el patrimonio alimentario de los ecuatorianos.

El sistema alimentario está sujeto a temas que se complementan con las actividades económicas, políticas, familiares y culturales, debido a que si se habla de patrimonio, se refiere a la herencia cultural y natural que aporta cada alimento, y al producto final que se consume.

La panificación tiene un gran valor histórico y riqueza culinaria, ya que tiene un legado que pasa de generación en generación y se edifica al compartir sus saberes y sabores. Además, debido al amplio abanico de recetas e ingredientes, cada región tiene su propia y autóctona gastronomía, por lo que se obtienen características particulares asociadas al placer de comer y beber bien.

La cultura alimentaria se enfoca en la suma de prácticas, conocimiento, consumo y producción de alimentos, ya que los alimentos listos para el consumo generan una importancia cultural e identitaria para un pueblo, por lo que se considera un patrimonio cultural alimentario.

La panificación ecuatoriana se caracteriza por los cambios sufridos en la estructura de la receta estándar, variación de los porcentajes panaderos de la materia prima y suplantación por otros materiales, debido a su costo y por la búsqueda de un producto accesible al presupuesto de los ecuatorianos, además existen panes que se han perdido con el transcurso del tiempo o están en proceso de terminar su existencia por varios factores. Debido a los cambios citados en la 
gastronomía y panificación de Ecuador, resulta un producto terminado que ha sido alterado y, por ende, empobrece la herencia cultural, patrimonial y social.

En el ámbito de la gastronomía, se manifiesta la necesidad de conocer los sitios que han perdurado en el tiempo y que han mantenido los productos de panificación; dichos lugares propician el desarrollo económico y sustento de un grupo de familias dedicado a la panificación artesanal.

\section{Metodología.}

La entrevista se define como la comunicación interpersonal establecida entre el investigador y el sujeto de estudio a fin de obtener respuestas verbales a los interrogantes planteados sobre el problema que se quiere investigar (Galán, 2009).

Dicha investigación debe obtener una información veraz de la población carchense, en especial de los panaderos de la ciudad, ya que la variedad de productos panificables tiene una historia y datos importantes en Carchi.

El método analítico consiste en el desglose de un macroartículo, que se descompone en sus partes o elementos para observar la causa, la naturaleza y el efecto (Limón, 2017). Se requiere indagar y plantear las causas de la pérdida de las costumbres y tradiciones en el tema puntual de la panadería de la provincia del Carchi.

La investigación de campo es través de la aplicación de la investigación cuantitativa, se procederá a la medición y cálculo, con el objetivo de buscar variables con referencia al caso de estudio, de tal forma que nos lleve a la obtención de resultados en base al control y predicción de la realidad para de esta forma exigir la confiablidad y validez en la medición, por medio del tratamiento de los datos se basa en la sistematización, el uso de cifras y la estadística.

\section{Resultados}

El patrimonio tiene por enfoque identificar aquellos productos elaborados, materia prima, valor tangible e intangible dentro de la sociedad, dichos rasgos distintivos mencionados, provocan sustentabilidad a nivel nacional e internacional dentro de una nación.

La revalorización del patrimonio alimentario local tiene por objetivo el reconocimiento, fortalecimiento de las identidades, por lo cual se garantiza una alimentación sana, nutritiva y culturalmente apropiada para los ecuatorianos. Es importante mencionar que la soberanía alimentaria mantiene fundamentos estrictos en la recuperación del rol de la sociedad para disipar en aspectos puntuales como: qué producir, como producir, donde producir, para quien producir (Patrimonio, 2013).

La panificación surge cuando los primeros panes fueron elaborados hace decenas de miles de años cuando los hombres que vivían de la caza, de la recolección y consumo de cereales. Inicialmente comían granos como la naturaleza se los ofrecía; más tarde comenzaron a machacarlos entre dos piedras para ablandarlos y posteriormente cocinarlos en agua haciendo 
una papilla. Esta papilla fue olvidad sobre fuego, se secó y dio origen a una galleta que se podía conservar por varios días, la que es considerada como" El primer pan ".

El pan "moderno" se elaboró en Egipto alrededor del año 3000 a.C. Según cuenta la historia, un artesano olvido hornear un pedazo de masa y al día siguiente, para evitar el castigo de su amo, mezclo esta masa que había fermentado con la nueva. El resultado fue que el pan era mucho mejor, había nacido el pan fermentado. El proceso se mejoró finalmente para el año 1000 a.C. cuando se logró aislar la levadura de la cerveza para agregarla directamente al pan.

Los egipcios mejoraran además los procesos agrícolas, desarrollando nuevas variedades de trigos, perfeccionaron los métodos de obtención de harinas e inventaron hornos donde podían cocerse varios panes a la vez.

Los griegos y luego los romanos tomaron las técnicas de panificación de Egipto y las llevaron exagerados niveles de sofisticación. Florecieron las panaderías, se diversificaron las masas, y se elaboraron más de 30 diferentes panes enriquecidos. De la mano de los romanos el pan se extendió por Europa y tomo matices distintos en cada región.

Durante la edad media la elaboración de panes retrocedió en sofisticación aunque se mejoraron los molinos y la agricultura. El pan convertido ya en el alimento básico de la población era también un símbolo de status, los panes blancos y enriquecidos eran para la clase alta y los oscuros elaborados con los desechos y los peores granos eran para los más pobres.

Con la llegada del Renacimiento, la modernidad y la revolución industrial, la molinería y la panadería se profesionalizaron, se industrializaron e incorporaron nuevas técnicas tecnologías y maquinaria para llegar a la panadería que hoy conocemos.

En el Ecuador inicia cuanto llega el trigo a través un Monge franciscano, nos enseña a cultivar, moler y obtener el la harina de trigo, esto se disipa a nivel de América. En la provincia del Carchi su panificación se desarrolló en base a los productos que se encontraban a disposición, así fue complementado la utilización de la harina, sal, agua y levadura, con producto autóctonos de la provincia del Carchi, a partir es esto nace el uso de la cuajada, queso amasado, manteca de chanco, panela entre otros.

La Cuajada es un producto lácteo elaborado a partir de leche, cuajo y suero, su obtención se basa en la coagulación de la leche a través del cuajo y suero, los cuales son escurridos levemente, para que retenga cierta cantidad de suero, por ende adquiera una textura suave cremosa y fluida.

El queso amasado Carchense es el queso no madurado obtenido de cuajada no cortada, de acidificación natural, molido, amasado, moldeado en moldes perforados y espolvoreado sal de consumo humano; desmenuzado manualmente, moldeado y prensado. Este producto lácteo es aplicado en los panes de la región, su expendio se da en los mercados de la zona. Jaramillo, C (2015). Estudio De Factibilidad Proyecto Queso Fresco Producido En Industrias Lácteas Toni S.A. Guayaquil. 
Manteca chancho: Materia grasa de origen animal, también llamado manteca de cerdo, producido por la cocción de los tejidos epidémicos del cerdo. Su color deber ser blanco, en estado sólido, presenta olor y sabor característico del producto.

Panela: Es un producto sólido que se obtiene por la evaporación de los jugos clarificados de la caña de azúcar, se obtiene del batido intenso de las mieles hasta alcanzar el punto de panela, su presentación final es en forma de bloques, polvo o granos ligeramente esféricos.

Ecuador tiene limitados y escasos escritos que permitan remontar la historia del pan. Se conoce a la panificación de forma histórica a nivel global y, en la actualidad, lo que sucede de forma científica.

Con la llegada de los españoles comienza el contacto con la primera espiga de trigo. Según Guillaumin, los religiosos europeos ayudaron a que los indígenas de México experimenten este arte de la panificación, del cultivo y otras actividades; por ello, se puede decir que la influencia europea ayudó a entender acerca de la diversa aplicación gastronómica de este cereal llamado trigo (Guillaumin, 2010).

Se encuentran documentos que hacen mención que en Ecuador inició el contacto con el trigo en el año de 1535. Existió este primer acercamiento por la llegada del Fray Jodoko Ricke. En las investigaciones de Nydia Vázquez de Fernández de Córdoba, en su libro Panes Tradicionales, nos relata la llegada del trigo con dicho personaje, ya que este es el inicio para que el trigo prolifere y, con el tiempo, se extienda por toda América (Vázquez, 1985).

Fray Jodoko Ricke llega al ya fundado San Francisco de Quito. Con el tiempo, el monje franciscano toma cariño a los indígenas, les demostró calidez y les ayudó intelectualmente enseñándoles a leer y escribir. Además les explicó el proceso de cultivo, cosecha y molienda del trigo; les instruyó para preparar los campos a través del arado con los bueyes y cómo tener un proceso adecuado en el manejo de las espigas. Más tarde, les indicó la elaboración del pan. Con el tiempo, el trigo se convirtió en un alimento importante en las provincias de la región interandina (Pachajoa, 2007).

Julio Pazos Barrera en su libro Sabores de la Memoria relata sobre la cocina y técnicas de cocción de los primeros pobladores de Ecuador. Además, menciona sobre la fabricación artesanal y el uso de tiestos, los cuales fueron utilizados en diferentes métodos de cocción, como las vasijas de barro, ollas esféricas de barro, los cuencos o compoteras, piedras de moler y hornos a leña, los cuales eran instrumentos originarios de nuestros antecesores. Todos estos utensilios suman una riqueza en cuanto a cultura para los ecuatorianos, porque fueron parte del ciclo de e la cocina y de la panadería, las cuales son parte fundamental para que ahora tenga trascendencia en la historia (Pazos, 2008).

\section{Historia de la panificación en la provincia del Carchi}

Existe un valor gastronómico en Ecuador y un sinnúmero de historias rescatadas en distintas etapas de su gastronomía. En cada rincón del país, encontraremos productos de panificación tradicional y ancestral que permanecen en la cultura del consumidor. 
Sin duda en la provincia de Carchi se ha recopilado mucha información histórica transcendental sobre cada uno de los barrios y, con ayuda del municipio, se ha registrado tanta historia de la cual podemos enunciar lo siguiente:

En Tulcán, se edificó un monumento denominado "El Pupo", el cual revela la historia, ya que este refleja la hospitalidad, franqueza, valentía e inventiva el espíritu trabajador, solidario y minguero. Una de las razones por la que se arraigó esta tradición es que soldados colombianos ingresaron a Ecuador con el objetivo de invadir, sin embargo fueron detenidos por soldados de la región norte. Los soldados carchenses regresaron victorioso de tal hazaña, sin embargo una líder de la oposición los insultó llamándolos indios roscas, "pupos", debido a que los soldados usaban un uniforme pequeño que les regaló el gobierno de la época, por lo tanto mostraban el pupo; debido a este antecedente los tulcaneños tomaron el sobrenombre de "pupos" (Prefectura del Carchi, 2016)

La Provincia de Carchi tiene un alto valor por su cultura, costumbres y tradiciones; tiene un amplio abanico de historia, cultura y fiestas. Cabe recalcar que las comunidades de la provincia de Carchi se originan de las culturas Cayapas, Pastos y Quillasingas.

Tulcán se caracteriza por sus festividades que se celebran en los meses de enero y marzo. Dichas celebraciones conmemoran el inicio de la festividad de la Cuaresma católica que reúne al pueblo carchense e inclusive a pobladores que no son de la provincia, como del sur de Colombia, particularmente de Ipiales. Las fiestas de Tulcán tienen la particularidad de sus pregones, disfraces distintivos y coloridos (Gobierno Provincial del Carchi, 2017).

La zona norteña cuenta con lugares turísticos. Los cantones que la conforman tienen lugares característicos como es el "El Cementerio de Tulcán" o "La gruta de la Paz". En cuanto a la flora carchense está "El bosque de los Arrayanes", "La reserva Ecológica del Ángel” (Ministerio de Turismo del Carchi, 2010).La región norte de Ecuador posee una gastronomía extensa, emblemática y tradicional. Cuenta con platillos como el hornado pastuso, cuy asado, llapingachos y la papa asada que es conocido como tripa mishki, nombre acunado por los carchenses.

En cuanto a las bebidas, se puede probar una bebida llamada tardón, la cual es originaria del cantón Mira, el morocho y el champús. Por último, se encuentra el quesillo con miel, originario de la parroquia de Tufiño (Ministerio de Turismo del Carchi, 2010). El queso amasado, originario de la provincia de Carchi, se obtiene debido a que pertenece a una zona caracterizada por la ganadería; tanto sus lácteos como sus quesos son productos referentes y apetecidos por los consumidores por su calidad y sabor.

La maquinaria antigua que se utiliza, y que mantienen la tradición en ciertos casos específicos, es el horno a leña. Se caracteriza por su forma y su fabricación en adobe, esta mezcla se la realizaba a base de lodo podrido y lana de borrego. (Vázquez, 1985).En cuanto a menaje, podemos mencionar el uso de la batea y artesa, instrumentos donde se mezclaba los ingredientes, se amasaba y sobaba el amasijo final. Este era construido por personas especialistas en su fabricación, era una especie de tina fabricada de madera (Vázquez, 1985). 
Entre los utensilios, se encuentra la pala, instrumento de cocina con un extremo plano y de madera, conectada con un palo de dos metros que facilitaba la extracción de los panes del horno. Se utilizaba el gancho, el que simulaba un garfio de metal acoplado a un palo de madera, este ayudaba a retirar el amasijo, sobre todo cuando la producción panadera era en grandes cantidades. Se usaban para trabajar las mesas de amasijo, su principal función era reposar los amasijos, después del procesos de amasado; a partir de esto, los panaderos boleaban o daban punto, previo a la segunda fermentación y horneado. Finalmente la escoba que tiene la particularidad de estar hecha a partir de un amarrado de hojas conectado a un extremo de un palo de madera con el propósito de limpiar los hornos (Vázquez, 1985).

La panificación carchense, lastimosamente, no tiene escritos fehacientes para poder presentarlos y darlos a conocer a los pobladores. Se determina que los ciudadanos de avanzada edad identifican panes típicos y de la gastronomía tienen el pleno conocimiento sobre productos que hoy en día tienen un declive en la provincia.

En el área panificadora se necesita recapitular y revalorizar el patrimonio que, en algunos casos, mantiene su identidad y es apreciado por los consumidores; pero, en otros casos, no es tradicional; por ende, va perdiendo identidad, transcendencia y valor ancestral en el mercado tulcaneño.

\section{Producto de panificación autóctona de Tulcán}

\section{Pan de harina de maíz}

Tiene su origen en la parroquia El Ángel, típico de las panaderías del sector e inclusive se lo puede adquirir en el parque de la Libertad. En cuanto a materia prima, este pan utiliza harina de maíz, típica de la región, el cual le aporta su característica particular, harina flor o harina de trigo, queso amasado de la región, mantequilla huevos y levadura. La harina de maíz aporta un sabor especial y su porcentaje panadero es de $50 \%$, el otro $50 \%$ corresponde a la harina flor o harina de trigo. Se hornea de manera tradicional en horno de leña y su precio estimado es de 20 centavos.

Para la elaboración del pan de maíz se realiza un pre fermento denominado "base", se lo realiza con doce horas de anticipación y consta de azúcar, agua y levadura. En el tiempo se reposo, la base entra en un proceso de autolisis, esto ayuda al pan a mejorar la elasticidad e incrementar la estructura del gluten.

En panificación, este tipo de amasijos corresponden a una clasificación denominada batidos, los cuales no han desarrollado gluten; tienen la particularidad de fermentar al ambiente y no son aptos para leudar en una cámara de leudo debido a su estructura, por lo que dicho reposo debe hacerse, como se mencionó, a temperatura ambiente para que actúe la levadura y al hornearse active los dos agentes leudantes: polvo de hornear y levadura fresca.

En Tulcán, la familia Narváez es una de las primeras que realizaban el pan de maíz artesanal; sin embargo, su producción diaria ha disminuido y actualmente tiene otras fuentes de ingreso como es el caso del expendio de hornado pastuso. 


\section{Pambazo}

El pambazo es un pan que se elabora con harina integral, harina de trigo, anís español, panela, esencia de vainilla, huevos levadura, mantequilla, entre los ingredientes más destacados, los cuales aportan sus características particulares.

Es un pan de origen colombo-ecuatoriano; sin embargo, en el Carchi el pambazo va perdiendo influencia en la población, va desapareciendo de a poco por dos motivos importante: por su costo y por su tamaño.

En Tulcán, el lugar donde todavía se fabrica este pan es en la panadería Abril, del licenciado Gonzalo Abril, quien manifiesta que una de las razones por la que se va perdiendo la costumbre se debe a que en el Carchi, zona de cultivo del trigo, no existían muchas molineras; a las que menciona son la molinera Los tres choros y la molinera de la familia que vivía atrás del cementerio, de la molienda de este trigo se obtenía la harina de afrecho para la realización del pambazo, ya que a este tipo de trigo molido se denominaba harina de pambazo.

\section{Pan de finados}

En Tulcán, la señora Polita Narváez Guerrero menciona que su negocio ha pasado por generaciones, tiene alrededor de cien años dando el servicio. Actualmente, el negocio complementa su administración con café y hornado.

Ella menciona que el pan se lo hacía con harina de trigo negro, ya que no existían harinas refinadas y que la materia prima inicial era manteca de cerdo, huevos y harina con salvado; estos ingredientes eran básicos para los panes de la zona.

Cabe recalcar que tras la ausencia de azúcar refinada, el pan se lo realizaba con miel. Su familia mantiene la receta de generación tras generación y la única diferencia es su mecanismo de cocción, el cual antiguamente se lo realizaba en un horno de leña y actualmente en un horno convencional, la principal razón por la que cambio el horno es debido al espacio que ocupaba, capacidad de cocción y practicidad.

La tradición se mantiene latente por dos razones: su negocio de panificación y restaurante, sumado a que, cada cierto tiempo que surge la oportunidad, planifican entre sus seres queridos reuniones que refuerzan la tradición panificadora entre su familia y seres queridos.La señora Polita Narváez recalca que una problemática es la globalización, ya que hace que el grupo de jóvenes tulcaneños, capten y se enriquezcan de lo que ven de afuera y aprecien en menor medida los productos tradicionales y autóctonos de Tulcán.

\section{Pan de cuajada}

El pan de cuajada es un pan que se elabora con harina de maíz, cuajada, polvo de hornear, mantequilla, harina de trigo, azúcar, leche y sal. Su característica principal es la cuajada, ya que dicho producto le aporta sabor; se lo obtiene a partir de leche, cuajo y suero. 
La familia Pantoja en Carchi mantiene el legado de la producción de panes artesanales, dicha panificadora está administrada por Luis Pantoja y Miriam Pantoja. Existe un detalle fundamental que ellos mantienen: el uso de bateas fomentan la utilización del método de cocción a través del horno de leña, manifiestan que ellos mantienen la tradición por 60 años.

\section{Pan de mote}

Pan típico de la región de Tulcán que se realizaba con mote, quesillo y sal. La señora Rosa Arteaga menciona que el procedimiento para elaborarlo consistía en pelar el mote con ceniza, lavarlo bien y dejarlo desaguar por un día, luego se lo molía en un molino de mano. A esta masa, con quesillo y sal, se la colocaba en hojas de achira en el horno de leña. Se lo puede encontrar en La Plaza Central del Buen Vivir de la ciudad de Tulcán todos los días.

\section{Rosquetes y Bizcochuelos}

En la parroquia Monte Olivo del cantón Bolívar, a 50 metros del templete de la Virgen de Fátima, patrona de los transportistas, está el local "La Delicia", donde se pueden encontrar las tradiciones gastronómicas de esta zona. Doña Lucila Verde soto desarrolló la idea del negocio hace cuarenta años e inició elaborando los tradicionales rosquetes y bizcochuelos. El negocio pasó a manos de su hijo Kléver Flores, quien es el responsable de que estas tradiciones sigan manteniéndose vivas. De acuerdo al nuevo propietario, la elaboración de los rosquetes y bizcochuelos en el lugar data de cientos de años e incluso se dice que fue un regalo especial en una visita que hiciera a esta tierra el Libertador Simón Bolívar (RCJC, 2015).

\section{Panes varios de la Provincia del Carchi}

En Mira, nos contaron también acerca de las paspas (pan de maíz y queso). "Se dice así por cómo se ponen los cachetes en los páramos, así mismo es el pan: áspero”. También se nos hizo mención acerca de "los socialistas", que denotan la coyuntura política vinculada a la tradición del pan (Villavicencio, 2005).

Son también características las panuchas y los rosquetes. Las primeras de una preparación parecida a la de los quimbolitos, "pero con la masita más seca y necesariamente en horno de leña". Para hacer los rosquetes "se bate los huevos (preferiblemente de campo) y se le pone una copa de puntas. Se les revuelve a punto de nieve las claras, lo mismo las yemas. Se mezcla con la harina para hacer la masa y se hacen las roscas con manteca de chancho" (Villavicencio, 2005).

\section{Discusión}

A través del desarrollo de este artículo y la aplicación de una encuesta en la muestra de la población, se determinó que existe una identidad en desarrollo en la panificación de la provincia del Carchi, debido a que existen panes que por falta de consumo, perdida de materia prima esencial, panificadoras artesanales en declive económico por competidores industrializados, precios bajos de las panificadoras industriales, pérdida de apego al valor patrimonial y cultural por parte de la sociedad. 
Tabla1: Consumo de panes tradicionales de la Provincia del Carchi.

Consumo de panes tradicionales de la Provincia del Carchi.

Muestra 383

Conocen la panificación del Carchi

No Conocen la panificación del Carchi 165

Consumo de panes típicos de la región de Mote, Pan de Maíz y Panuchas.

Lugares donde se realizan panificación típica Panadería Abril, Panadería Narváez, Panadería la Delicia, de la provincia del Carchi.

Panadería de la Sra. Arteaga, Panadería de la Familia Pantoja.

Pan de mote $46.2 \%$.

Pan de cuajada 45.7

Pan de Maíz $50.9 \%$

Porcentaje de Consumo de Panes en la Pambazo 39.7\%

Provincia del Carchi.

Pan de Paspas 5.7

Otros $10.9 \% *$ (panuchas, rosquetes y pan de paspas.

Elaborado por: Daniel Fierro 2018

A pesar de la problemática mencionada debido a la pérdida de identidad por los factores mencionados, existen panes que siguen vigentes, forman parte de la cultura y dieta del pueblo carchense, sin embargo la memoria de consumidor acaparan unos más que otros.

Se menciona a través del análisis, panes tales como Pan de Maíz, Pan de Cuajada y Pan de mote, se encuentran vigentes, forman parte de la vida del pueblo carchense, sin embargo y a través de la consulta, panes como Pan de Pambazo, Paspas y Panuchas, existen una pérdida de identidad gastronómica, por lo que sería apropiado rescatar y revalorizar en dichos panes para generar una cultura que valore procesos, técnicas, recetas y por ende generar identidad gastronómica.

Las panaderías de la zona tienden a redondear sus réditos económicos a través de la venta de productos sustitutos, sea mediante el expendio de hornado pastuso, helados, etc. En tanto, el equipamiento y utensilios artesanales tienden a desaparecer, la predisposición de los panificadores artesanales es modernizarse, las razones principales por el abandono, son el espacio que ocupan y la capacidad de producción de los hornos de leña.

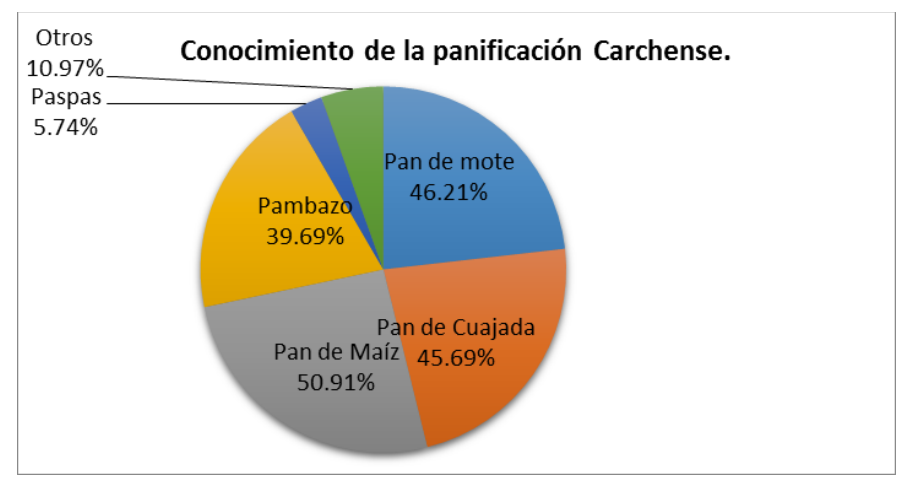

Gráfico 1. Conocimiento de la panificación del Carchi. 
Figura 1: Conocimiento de la panificación del Carchi. Realizado por: Milton Silvio Haro Peña.

\section{Conclusiones}

La panificación artesanal del Carchi tiene la dificultad de mantener su tradición, identidad y cultura debido a la perdida de ingredientes tradicionales, como es el caso del pan Pambazo, ya que la producción de harina de pambazo, está desapareciendo por temas como la falta de molinos artesanales en la región y cultivo del trigo autóctono de la región.

Las panificadoras artesanales que mantienen su legado por más de 60 años, se han adaptado a las necesidades de equipamiento y utensilios modernos, pocos han perdurado en el tiempo manteniendo su identidad y conservación de las técnicas en procesos de panificación artesanal.

Las recetas han mantenidos su tradición en el uso de materia prima de la zona como es el caso del uso del quesillo, cuajada, harina de maíz, etc., sin embargo para abaratar costos y ser competitivos en el mercado carchense, usan materias grasa comercial, azúcar refinado y harina de trigo comerciales.

Los panes del Carchi, si bien unos panes están en la mente del consumidor y están a la venta a diario como es el caso del Pan de Maíz, pan de Mote y Cuajada, existe también panes que van perdiendo campo comercial y tienden a la desaparición tales como pan Paspas, panuchas, etc.

El grupo vulnerable según la investigación se determina que los jóvenes entre 18 y 25 años, muestran una tendencia a desvalorizar la tradición gastronómica de la zona y se identifica como una población que se inclina por modas, globalización y valorar lo que ocurre fuera de su provincia.

\section{Agradecimiento}

A la Universidad Tecnológica Equinoccial por ser parte de la formación integra como docente, a las autoridades académicas de la Facultad de Hospitalidad y Servicio por ofrecer los lineamientos adecuados para la conclusión del artículo.

A nuestros hijos y familias que son la motivación diaria para el cumplimiento de nuestra labor como docentes de Universidad Tecnológica Equinoccial.

A Milton Silvio Haro Peña por la colaboración con datos oportunos sobre los temas tratados en la investigación del artículo del Carchi.

\section{Bibliografía}

Alejandra Rosero. (2015). Estudio investigativo del mote, analisis de sus propiedades y elaboracion de alternativas para la cocina ecuatoriana. Quito. 
Aviles, E. (n.d.). Enciclopedia del Ecuador. Retrieved Agosto 21, 2017, from http://www.enciclopediadelecuador.com/historia-del-ecuador/gran-colombia/

Bernal, C. (2010). Metodología de la investigación (administración, economía, humanidades y ciencias sociales). Bogotá: Pearson.

EcuaRed. (2009). Provincia del Carchi. Retrieved Agosto 23, 2017, from https://www.ecured.cu/Provincia_de_Carchi

El telegrafo. (2011, Septiembre 1). El telegrafo. El telegrafo, p. 1.

Galan, M. (2009, Mayo 29). Metodologia de la Investigacion. Retrieved from http://manuelgalan.blogspot.com/2009/05/la-entrevista-en-investigacion.html

Gobierno Provincial del Carchi. (2017). Carchi te enamora. Retrieved Agosto 22, 2017, from http://www.carchi.gob.ec/turistico/index.php/festividades

Guillaumin, M. D. (2010, Noviembre). Historia del pan en Mexico. Retrieved Julio 11, 2017, from http://www.historiacocina.com/paises/articulos/mexico/panmexico.htm

INEC. (2011, Septiembre 1). Instituto Nacional de Estadisticas y Censos. Retrieved Octubre 25, 2017, from Poblacion Demografica: http://www.ecuadorencifras.gob.ec/wpcontent/descargas/Manu-lateral/Resultados-provinciales/carchi.pdf

Limon, R. (2007). Eumend. Retrieved from Enciclopedia Virtual: http://www.eumed.net/librosgratis/2007a/257/7.1.htm

Ministerio de Turismo del Carchi. (2010). Carchi Bello. Retrieved Julio 03, 2017, from http://carchibello.webnode.es/:\%20de\%20http://carchibello.webnode.es/tradiciones/

Pachajoa, M. (2007, Julio 26). Fray Jodoko Ricke . Retrieved Agosto 22, 2017, from http://mariopbe.com/a7jo.htm

Patrimonio, M. d. (2013). Patrimonio alimentario. El Telëgrafo, 2-5.

Pazos, J. (2008). El sabor de la memoria: Historia de la cocina quiteña. Quito: FONSAL.

Prefectura del Carchi. (2016). Prefectura del Carchi. Retrieved Agosto 22, 2017, from http://www.carchi.gob.ec/2016f/index.php/historia-del-pupo.html

RCJC. (2015, 10 12). Las tradiciones de Bolívar no pierden vigencia. La Hora, 1.

Vazquez, N. (1985). Panes Tradicionales. Cuenca.

Villavicencio., M. V. (2005, Octubre). La Cultura Popular del Ecuador. 450. 